\title{
Fluid-repellent surgical mask (FRSM) fit - one size does not fit all
}

\author{
Authors: Lorna Russell, ${ }^{\mathrm{A}}$ Leyla Campani, ${ }^{\mathrm{A}}$ Jack Jones $^{\mathrm{A}}$ and Brendan Healy
}

\section{Background}

Fluid-repellent surgical masks (FRSMs) are recommended by the UK government for healthcare workers as personal protective equipment (PPE) against SARS-COV-2. UK Infection Prevention and Control (IPC) national guidelines states that 'masks must be well-fitting and fit for purpose, fully covering the mouth and nose'.

\section{Aim}

To review the fit of the FRSM supplied to the NHS front line workers against the national IPC guidelines and, through reaudit, assess for improvements in fit with FRSM worn with a plastic strap (intervention A) and FFP3 mask (intervention B).

\section{Method}

A three-part closed-loop audit was carried out comprising controlled observation, observation in the clinical area and questionnaire. Re-audit was carried out following interventions $A$ and $B$.

\section{Results}

FRSMs slipped below the nose in $43 \%$ and below the mouth of $10 \%$ of participants during the controlled observation and below the nose (above or below the mouth) in $30 \%$ of staff in the clinical area. No masks slipped below the nose or mouth with intervention A or B. $\mathbf{8 6 \%}$ of participants reported touching the FRSM to keep it in the correct position and $66 \%$ reported touching the FFP3.

\section{Conclusion}

The current supply of FRSMs are poorly fitting for many users and do not meet the UK IPC guideline standard. These issues were not evident when worn with a plastic strap or with FFP3 masks.

KEYWORDS: COVID-19, infection prevention, infection control, personal protective equipment, fluid-repellant surgical mask

DOI: $10.7861 /$ clinmed.2021-0054

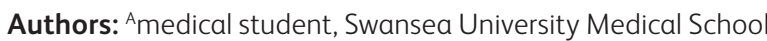
Swansea, UK; ${ }^{B}$ Consultant in microbiology and infectious diseases, Public Health Wales Microbiology, Swansea and Cardiff, UK and Department of Medical Microbiology, Morriston Hospital, Swansea, UK

\section{Introduction}

SARS-CoV- 2 is the novel coronavirus that causes coronavirus disease 2019 (COVID-19). First detected in December 2019, it subsequently spread rapidly, leading to the declaration of a pandemic by WHO in March 2020.?

SARS-CoV-2 is primarily transmitted through close contact with an infected individual. Whether predominately via respiratory droplet or aerosol (a matter of conjecture), transmission can occur through routine social contact during tidal breathing, speaking and coughing. ${ }^{1}$ The relative contribution of fomite spread is also uncertain but it is likely responsible for at least some transmission. Fomite transmission from contaminated personal protective equipment (PPE) is of potential concern and important in the healthcare setting. ${ }^{1-3}$ As worldwide cases of COVID-19 are currently over 120 million and deaths surpass 2.7 million, it is vital to break the chain of infection wherever possible as each transmission event has the potential to generate a new chain of infections. ${ }^{1,2,4,5}$

Fluid-repellent surgical masks (FRSMs) are designed to be worn over the mouth and nose to protect the mucous membranes from splashes and infectious droplets. ${ }^{2,4,6}$ The UK Government recommends them for use during contact with any patient regardless of their COVID-19 status. ${ }^{6}$ They are constructed to a standard that specifies construction, design, performance requirements and test methods (EN14683:2019). The masks are tested for bacterial filtration efficiency, breathability, splash resistance, microbial cleanliness (bioburden) and biocompatibility. The level of efficiency offered by a mask depends on a number of factors including filtration efficiency, material used and fit, which varies considerably with design. Most suppliers have a 'one size fits all' approach. The UK Infection Prevention and Control (IPC) national guidelines states 'the masks must be well-fitting and fit for purpose, fully covering the mouth and nose ${ }^{\prime 6}$ as otherwise their effectiveness is reduced. ${ }^{2,6}$ Furthermore, they stipulate that 'FRSMs should not be touched once put on or allowed to dangle around the neck. ${ }^{6}$ A previous report by O'Dowd et al noted a poor fit factor with FRSMs, suggesting potential compromise in protective efficacy. ${ }^{2}$ Healthcare workers (HCWs) are at greatly increased risk of infection with COVID19. ${ }^{4}$ Risks include PPE malfunction, failure to use PPE and incorrect use of PPE. ${ }^{2,7,8,9}$ Focussing on all aspects of the preventative measures deployed is crucial in reducing risk. In this paper, we investigate the issue of mask fitting as one critical component of these measures.

The aim of our audit was to review the fit of FRSMs supplied to patient-facing workers in the NHS and through re-audit, assess for improvements in fit with FRSMs worn with a plastic strap (intervention A) and with FFP3 masks (intervention B). 


\section{Method}

A three-part audit of mask fitting designed to obtain triangulation of results in relation to mask fit, comprising:

> a questionnaire (100 participants)

$>$ controlled observation of mask fit (30 participants observed over a total of 600 minutes)

$>$ observation of mask fit and mask etiquette in the clinical area (30 participants).

The audit comprised of staff members working on acute wards (designated COVID-19 and standard wards) across two hospital sites within a health board in South Wales in December 2020. Reaudit took place in January 2021. Participants in each section of the audit were selected at random from the personnel on the ward at the time the audit was carried out. Participation in one part of the audit did not exclude participation in another part of the audit and as such there is some overlap of participants between the groups. Verbal consent was gained for participation on the questionnaire and the controlled audit. Permission to conduct the ward area observation was obtained from the ward manager. All participants were selected at random. Audits of this type form part of routine infection, prevention and control practice. To minimise the Hawthorne effect, ${ }^{10}$ staff were not informed of the purpose of the study until after the completion of the observations. The three parts of the audit were carried out independently of one another. The intention was to increase the strength of any finding by demonstrating its presence or absence in three separate testing strategies. A mask was considered to have slipped below the nose if both nostrils were visible and below the mouth if the whole upper lip (and thereby the mouth if the mouth was open) was visible.

For the controlled observation of mask fit, participants were asked to read aloud one of three selected poems (the majority read 'Twas the night before Christmas'). The process was timed and if the mask slipped below the nose or mouth, the time taken for this to occur was recorded. Participants were instructed not to adjust the mask for the duration of the reading. Time taken to complete the reading was approximately 3 minutes.

For the observation of mask fit and behaviour in the clinical area, participants were surreptitiously observed for 20 minutes, recording each time any part of the mask was touched and if the mask fell below the nose or mouth. The part of the mask touched and the perceived reasons why the mask was touched (as subjectively assessed by the observer) was documented.

The re-audit followed the same methodology as the original audit. Two separate interventions were assessed:

> Intervention A: A plastic wrap around band was worn on the back of the head with FRSM ear loops hooked onto it questionnaire not performed as the bands are not routinely used.

> Intervention B: FFP3 masks - carried out in ITU and clinical areas where FFP3 masks are used in everyday practice. All participants had passed the Health board's Qualitative FFP3 mask fitting assessment.

\section{Results}

Initial audit

The FRSMs being used on the wards at this time were standard issue pandemic stock disposable medical mask executive standard BS EN 14683:2019 that use a nose clip and ear loop design.

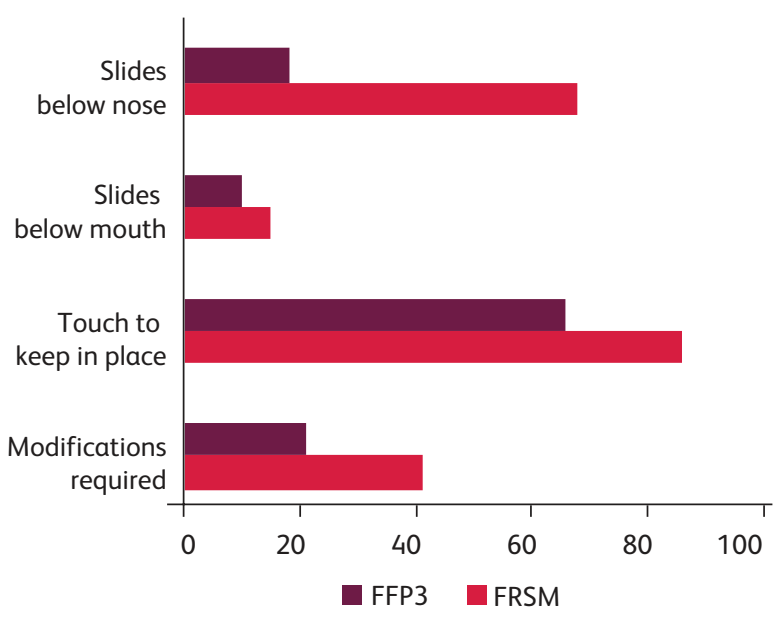

Fig 1. A cluster bar chart comparing the percentages of healthcare workers who report issues with the FRSM and the FFP3 mask which could compromise their efficacy.

\section{Questionnaire}

$68 \%$ of HCWs reported the FRSM sliding below their nose and 15\% below their mouth when worn as supplied (Fig 1). $86 \%$ reported having to touch the mask to keep it in the correct position. When asked which part of the FRSM they touch most frequently and why, 78 reported touching the nose bridge to pinch it, 49 the front of the mask to reposition it, 24 the ear loops, 11 the nose bridge for other reasons, five the front of the mask for other reasons and 20 for other reasons. $41 \%$ reported applying modifications or adjusting the mask when wearing it ( $49 \%$ of women vs $20 \%$ of men); 26 twisting the ear loops; 14 wearing a mask strap; eight twisting ear loops and flattening the mask across face; eight tying a knot in the ear loops at the mask end and four making other adjustments. 55\% reported that they do not feel that the FRSM offers sufficient protection from COVID-19 (Fig 2).

\section{Controlled observation}

The mask slipped in 13 participants ( $43 \%$ ), slipping below the nose only in 10 participants (33\%), and below the mouth in a further three $(10 \%)$ (Table 1). In six participants $(20 \%)$ the mask slipped below the

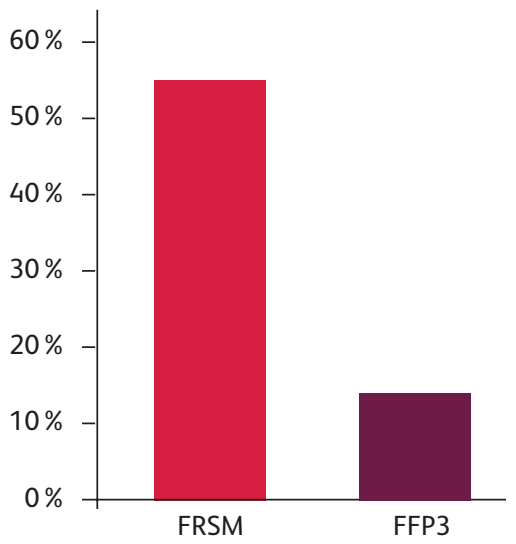

Fig 2. Visual representation of the percentage of healthcare workers who feel the masks do not offer sufficient protection from COVID-19 in the work that they do. 
Table 1. Percentage of participants in controlled observation who maintained full covering of nose/mouth in initial audit and following interventions

\begin{tabular}{lllllll} 
& \multicolumn{2}{l}{$\begin{array}{l}\text { Initial audit - } \\
\end{array}$} & & \multicolumn{2}{l}{ Intervention A - } & \multicolumn{2}{l}{ Intervention B - } \\
& FRSM only & & FRSM with plastic strap & \multicolumn{2}{l}{ FFP3 mask } \\
& $\mathbf{n}$ & \% Achieved & $\mathbf{n}$ & \% Achieved & n & \% Achieved \\
Fully covering nose & 30 & $57 \%$ & 30 & $100 \%$ & 30 & $100 \%$ \\
Fully covering the mouth & 30 & $90 \%$ & 30 & $100 \%$ & 30 & $100 \%$
\end{tabular}

nose in under one minute, in five between one to two minutes (17\%) and in two after two minutes (7\%). The mean time for a mask to slip below the nose was 1.25 minutes (SD: 0.69) (Fig 3).

\section{Observation in the clinical area}

In total 213 mask touches were recorded, a mean of one touch every 2 minutes and 48 seconds. Of these, 88 (41.3\%) were likely due to poor mask fit requiring pinching of the nose (42), adjusting the ear loops (10), and readjusting the mask after sliding below nose or nose and mouth (36). The remaining 125 (58.7\%) were recorded as 'other' and likely related to poor mask etiquette (Fig 4). Of the 30 participants, 25 (83\%) touched their mask at least once, $16(53 \%)$ touched it 5-10 times and eight (27\%) touched it $>11$ times. The mask was observed to have slipped below the nose or mouth at least once in nine (30\%) participants. In six $(20 \%)$ of these the mask slipped below the nose or mouth on multiple occasions.

\section{Re-audit}

\section{Questionnaire}

In intervention B (FFP3 mask), 18\% reported the mask sliding below their nose (cf 68\%), 10\% below their mouth (cf 15\%), 21\% reported applying modifications ( $28 \%$ of women vs $3.5 \%$ of men) and $66 \%$ reported having to touch the mask to keep it in the correct position - touching the nose bridge to pinch it was reported as the most common reason (54\%) (Fig 1). $15 \%$ of respondents felt the mask did not offer sufficient protection from COVID-19 (Fig 2).

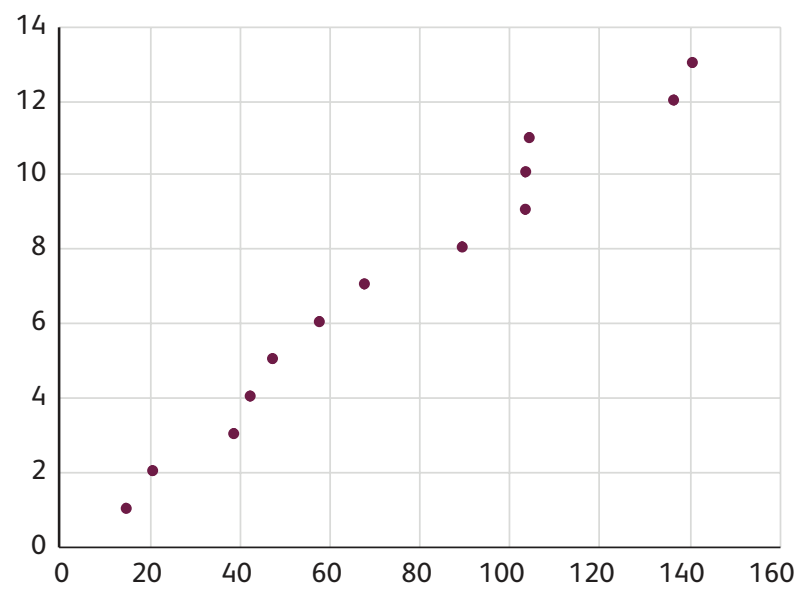

Fig 3. Scatter graph displaying the specific time at which the FRSM slipped to below the nose in 13 out of the 30 participants during the controlled observation.

\section{Controlled observation of mask fit}

Masks did not slip below the nose or mouth of any of the 30 participants in either intervention A or B (Table 1).

Observation of mask fit and behaviour in the clinical area Intervention A: A total of 46 mask touches were recorded, 32 likely due to poor fit and 14 'other' (Fig 4). Seventeen (56\%) participants did not touch the mask during the observation, 12 (40\%) touched it $0-5$ times and two $(6.6 \%)$ people touched it 5-11 times.

Intervention B: A total of 31 touches were recorded, 20 likely to poor fit and 11 'other'. Sixteen (53\%) participants did not touch the mask during the observation, $14(46 \%)$ touched the mask $0-5$ times. The highest number of touches by one participant was four. Masks did not slip below the mouth or nose in either intervention arm.

\section{Discussion}

This study highlights concerns in relation to the fit of FRSMs (IIR) as currently provided. Poorly fitting masks create risks in the following ways:

> The protective efficacy of the mask is reduced.

$>$ The mask needs to be touched and adjusted frequently to keep it in the correct position.

> Modifications are required to keep the mask in the correct position. These modifications may compromise mask efficacy and breach the recommendations within the UK IPC national guidelines, which state 'manufacturers' instructions must be followed to ensure effective fit and protection'.

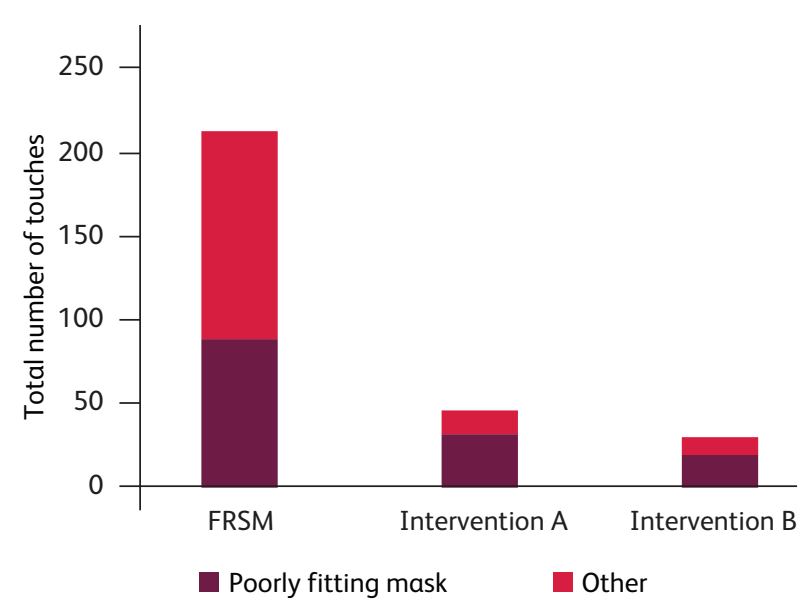

Fig 4. Bar chart comparing the total number of touches observed during the observation in a clinical setting in each cycle of the audit. 
As such, the poor fit of the mask has the potential to increase risk of transmission through both airborne and fomite routes.

Although the FRSMs are designed with protective features, ie fluid resistance and malleable nose bridge, ${ }^{6}$ this report supports the notion that the current masks being supplied do not meet the specification of the UK IPC national guidelines, which state, 'the masks must be well-fitting and fit for purpose, fully covering the mouth and nose'.

This audit demonstrates that a 'one size fits all' approach with FRSMs is ineffective and that the FRSMs provide a poor fit for many HCWs. Most strikingly, $86 \%$ of participants report the need to touch the FRSM to keep it in the correct position, the masks slipped below the nose in $43 \%$ and below the mouth in $10 \%$ of participants during the controlled observation and masks slipping below the nose (+/- the mouth) was observed to affect $30 \%$ of staff in the clinical area. Mask fit was improved in both intervention arms and masks did not slip below the noses or mouth during FFP3 mask use or when FRSM was worn with a plastic strap.

Of note, $49 \%$ of women reported having to make modifications or adjustments to the FRSM when wearing it compared to $20 \%$ of men. Gender differences in the fit of personal protective equipment have been highlighted elsewhere and should be considered in the design. ${ }^{9}$ Reported need to make modifications was reduced with the FFP3 masks - women $28 \%$ and men $3.5 \%$.

$55 \%$ of participants reported that they do not feel that the FRSM offers significant protection. A recent Cochrane review found that if HCW trusted the efficacy of the PPE, adherence towards IPC guidance is greater. ${ }^{8}$ Only $14 \%$ reported a lack of confidence in FFP3 masks.

The total number of mask touches in the clinical area reduced from 213 to 46 (intervention A) and 31 (intervention B), with 56\% of participants (intervention A) and 53\% (intervention B) not touching their mask at all (cf 17\% FRSM alone). In intervention B no participant touched their mask more than five times (cf $27 \%$ of participants touching their mask $>11$ times FRSM alone).

In the original audit, staff often touched their FRSM for reasons of perceived poor mask etiquette (58.7\%) often related to habitual behaviours, such as: touching face, leaning on hands, or lowering mask to speak. Interestingly these behaviours were reduced when the mask fit improved suggesting that not all the touches are purely habitual. Regardless, a need for continued education on the correct usage of PPE is required.

While plastic straps improved the mask fit, they do provide a logistical challenge if deployed for routine use as they are supplied separately from the mask. If unavailable for any reason, the performance of the mask will be compromised in line with the original audit. As well as additional cost, they are also not single use and introduce issues related to cleaning, reuse and associated risk of fomite transmission.

\section{Limitations}

Limitations of this audit include small participant numbers. However, the prevalence of observed problems mean that a larger audit is unlikely to alter the findings significantly. In addition, given the significance and importance of the findings it was decided not to extend the audit any further but rather report the finding so that action could be taken. The observational audit of staff during clinical practice includes some subjective interpretation on the reason for the mask being touched. This part of the audit is vulnerable to misinterpretation and observer variation. However, even with significant inter-observer variation in interpretation it would not alter the overall findings of the audit.

\section{Conclusion}

This report demonstrates that the current supply of FRSMs in our area have a poor fit for many users and do not meet the standards of the UK Infection Prevention and Control (IPC) national guidelines thereby providing a potential transmission risk for staff through droplet, aerosol and fomite transmission. Fit is improved by using a plastic strap or an FFP3 mask. Evidence from this study should be used to prompt a reconsideration of the infection, prevention and control guidelines, PPE supply and to drive enhancements in PPE design to improve protection for HCW's on the front line. Further research into the design and specification of masks, including potential gender and racial differences, is required to ensure protection in the real-world setting is delivered.

\section{References}

1 BMJ Best Practice. Coronavirus disease 2019 (COVID 19). https:// bestpractice.bmj.com/topics/en-gb/3000201.

2 O'Dowd K, Nair KM, Forouzandeh P et al. Face masks and respirators in the fight against the COVID-19 pandemic: A review of current materials, advances and future perspectives. Materials (Basel) 2020;13:3363.

3 Chughtai AA, Stelzer-Braid S, Rawlinson W et al. Contamination by respiratory viruses on outer surface of medical masks used by hospital healthcare workers. BMC Infect Dis 2019;19:491.

4 World Health Organization. Rational use of personal protective equipment (PPE) for coronavirus disease (COVID 19). Interim guidance. WHO, 2020. Available from https://apps.who.int/iris/ bitstream/handle/10665/331498/WHO-2019-nCoV-IPCPPE_use2020.2-eng.pdf

5 European Centre for Disease Prevention and Control. COVID-19 situation update worldwide, as of 14 December 2020. ECDC, 2020. www.ecdc.europa.eu/en/geographical-distribution-2019-ncov-cases

6 Public Health England. COVID-19: Guidance for maintaining services within health and care settings: infection prevention and control recommendations. PHE, 2020. https://assets.publishing.service.gov.uk/ government/uploads/system/uploads/attachment_data/file/910885/ COVID-19_Infection_prevention_and_control_guidance_FINAL_ PDF_20082020.pdf.

7 Avo C, Cawthorne K-R, Walters J, Healy B. An observational study to identify types of personal protective equipment breaches on inpatient wards. J Hosp Infect 2020;106:208-10.

8 Houghton C, Meskell P, Delaney H et al. Barriers and facilitators to healthcare workers' adherence with infection prevention and control (IPC) guidelines for respiratory infectious diseases: a rapid qualitative evidence synthesis. Cochrane Database Syst Rev 2020;4:CD013582.

9 Ascott A, Crowest P, de Sausmarez E, Khan M, Chakladar A. Respiratory personal protective equipment for healthcare workers: impact of sex differences on respirator fit test results (published online ahead of print, 2020 Oct 21). Br J Anaesth 2020;126:e48-9.

10 Franke RH, Kaul JD. The Hawthorne experiments: First statistical interpretation. Am Sociol Rev 1978;43:623.

Address for correspondence: Dr Brendan Healy, Public Health Wales Microbiology (Cardiff and Swansea), University Hospital of Wales, Heath Park, Cardiff CF14 4XW, UK. Email: brendan.healy@wales.nhs.uk 